This item was submitted to Loughborough's Research Repository by the author.

Items in Figshare are protected by copyright, with all rights reserved, unless otherwise indicated.

\title{
A theoretical study of intrinsic point defects and defect clusters in magnesium aluminate spinel
}

PLEASE CITE THE PUBLISHED VERSION

http://dx.doi.org/10.1088/0953-8984/21/27/275406

PUBLISHER

(C) IOP PUBLISHING LTD

VERSION

AM (Accepted Manuscript)

\section{PUBLISHER STATEMENT}

This work is made available according to the conditions of the Creative Commons Attribution-NonCommercialNoDerivatives 4.0 International (CC BY-NC-ND 4.0) licence. Full details of this licence are available at: https://creativecommons.org/licenses/by-nc-nd/4.0/

\section{LICENCE}

CC BY-NC-ND 4.0

\section{REPOSITORY RECORD}

Gilbert, Christopher A., Roger Smith, Steven D. Kenny, S.T. Murphy, R.W. Grimes, and J.A. Ball. 2019. "A Theoretical Study of Intrinsic Point Defects and Defect Clusters in Magnesium Aluminate Spinel”. figshare. https://hdl.handle.net/2134/19331. 


\title{
A theoretical study of intrinsic point defects and defect clusters in magnesium aluminate spinel
}

\author{
C. A. Gilbert, R. Smith, and S. D. Kenny \\ Department of Mathematical Sciences, \\ Loughborough University, Loughborough, LE11 3TU.
S. T. Murphy, R. W. Grimes, and J. A. Ball
Prince Consort Road, London, SW7 2BP. \\ Department of Materials, Imperial College,
}

\begin{abstract}
Point and small cluster defects in magnesium aluminate spinel have been studied from a first principles viewpoint. Typical point defects that occur during collision cascade simulations are cation anti-site defects, which have a small formation energy and are very stable, $\mathrm{O}$ and $\mathrm{Mg}$ split interstitials and vacancies. Isolated $\mathrm{Al}$ interstitials were found to be energetically unfavourable but could occur as part of a split $\mathrm{Mg}$-Al pair or as a three atom-three vacancy Al 'ring' defect, previously observed in collision cascades using empirical potentials. The structure and energetics of the defects were investigated using density functional theory (DFT) and the results compared to simulations using empirical fixed-charge potentials. Each point defect was studied in a variety of supercell sizes in order to ensure convergence. It was found that empirical potential simulations significantly overestimate formation energies, but that the type and relative stability of the defects are well-predicted by the empirical potentials both for point defects and small defect clusters.
\end{abstract}

PACS numbers: 31.15.Ew, 61.72.-y, 61.82.Ms

Keywords: Magnesium aluminate spinel; defects; DFT

\section{INTRODUCTION}

Magnesium aluminate spinel $\left(\mathrm{MgAl}_{2} \mathrm{O}_{4}\right)$ is one of many crystalline oxide compounds upon which radiation damage experiments have been performed and which has been the subject of recent cascade simulation studies ${ }^{1-3}$. The motivation for this is due, at least in part, to the need to derive new materials with enhanced radiation resistance. Experimental work on spinel, carried out over a number of years, has shown that under neutron bombardment, a large number of cation anti-site defects ${ }^{4}$ are produced and that in addition stable point defects, preceding either amorphisation or a transformation to a rocksalt structure, could be formed under high dose inert gas ion bombardment ${ }^{5-7}$. Classical molecular dynamics simulations that examined the ballistic phase of the cascade ${ }^{1,2}$ also concluded that the predominant defects remaining after this phase were cation anti-site defects but that split $\mathrm{Mg}$ and $\mathrm{O}$ interstitial defects could occur orientated in $<110>$ directions. In addition, split $\mathrm{Mg}$ - $\mathrm{Al}$ interstitials formed, centred on an $\mathrm{Mg}$ site and also a three atom-three vacancy Al 'ring' defect. Isolated vacancies or vacancy clusters were also observed. Isolated aluminium interstitial defects were not observed and when following the defect evolution over longer times the split $\mathrm{Mg}-\mathrm{Al}$ interstitials decayed to a split $\mathrm{Mg}-\mathrm{Mg}$ interstitial and an $\mathrm{Al}$ antisite defect. Larger defects involving $\mathrm{Al}$ interstitials were also rare. These results derived using empirical potentials, while in agreement with the available experimental evidence, do not necessarily give the most accurate model of the defect energetics. It is therefore of interest to investigate point defects in magnesium aluminate spinel using density functional theory (DFT).

Unlike the classical empirical potential simulations, only a limited amount of work has previously been carried out on spinel using a first principles approach. Much previous ab-initio work has focussed on cation disordering and inversion within the structure. Work by Warren et al. ${ }^{8}$ compared ab-initio results computed using both the local density approximation (LDA) and the generalised gradient approximation (GGA) with a Monte Carlo simulation. Their work involved exchanging varying numbers of magnesium and aluminium atoms within the system and computing the energy differences between each simulation. Using results of simulations of systems containing varying degrees of disorder within spinel, Ball et al. ${ }^{9}$ studied the effects of this on the computed lattice constant. In addition, they also concluded, through comparisons between DFT and pair potential computations, that LDA represents the experimental behaviour better than GGA. DFT calculations were also carried out by Moriwake et al. ${ }^{10}$ in an investigation of aluminium vacancies. These authors conclude that the formation energy of an aluminium vacancy can be as large as $13 \mathrm{eV}$.

In previous work ${ }^{11}$, we studied defects and transition barriers in magnesium oxide from a first principles perspective and compared our results with calculations using empirical potentials. These results showed fairly good agreement between the formation energies of the Schottky defects, the defect geometries and the transition barriers of isolated 
defects but there were some differences with the di-interstials. Here, a similar analysis has been carried out on $\mathrm{MgAl}_{2} \mathrm{O}_{4}$ but this time concentrating on geometries and formation energies of defects rather than transition energy barriers. We utilise a DFT framework to compute the structures and energetics of the defects observed in collision cascades together with possible alternative structures. In addition, instead of considering isolated vacancies alone, as in the study by Moriwake et al. ${ }^{10}$, we investigate the energetics of small cluster defect structures and defect reactions. For the empirical potentials a model both with shells and without shells is considered. The shell model is generally thought to be more accurate while the model without shells is usually employed in dynamical cascade simulations.

\section{METHODOLOGY}

This study of the energetics and relaxed structures of defects within spinel has been carried out using PLATO ${ }^{12}$, a DFT code ${ }^{13,14}$, with the exchange-correlation being described using the LDA ${ }^{15}$. Pseudopotentials for magnesium, oxygen and aluminium have been taken from the work of Hartwigsen, Goedecker and Hutter ${ }^{16}$ and basis sets were created using localised, numerical orbitals. A semicore basis set was used for magnesium, with $s, p$ orbitals taken from the neutral atom and $s, p$ and $d$ orbitals taken from taken from the doubly positively charged atom. A non-semicore basis set was used for aluminium and oxygen using the same approach. Using a suitable k-point mesh, and performing numerical integrals over an atom-centred grid, our basis set estimated the lattice constant and bulk modulus of spinel to be $8.016 \AA$ and 2.01 Mbar respectively. These values are in good agreement with previously reported experimental values of $8.089 \AA$ and 1.96 Mbar. It is noted that Gupta et al. ${ }^{17}$ computed a lattice constant of $8.083 \AA$ using DFT with LDA. We found that, upon relaxation of a perfect system, our estimate of the $u$ parameter (which is a measure of the oxygen dilation) was 0.389 (only 0.001 larger than the reported experimental value for $\mathrm{MgAl}_{2} \mathrm{O}_{4}$ ). $\mathrm{Supercells}_{\mathrm{s}}$ were created using the methodology described in our previous work ${ }^{11}$ similar to that implemented in the computation tool CRYSTAL ${ }^{18}$. The methodology is utilised in order to maximise the separation distance between the defect and its periodic repeats, whilst minimising the number of atoms in the supercell. Supercells were created which contained 56, 112, 168 and 224 lattice sites through a repeat of the 14 atom primitive cell ${ }^{19}$. Keeping the number of lattice sites in the supercell fixed, it was possible to obtain the most efficient shape of supercell for each cell size and defect configuration.

Previous empirical potential simulations used a fixed charge model with the charge of +2 on a magnesium atoms, -2 on oxygen and +3 on aluminium. In our DFT calculations, we have added and removed electrons to the defective supercells in order to replicate charged defects. Tests were performed to ensure that the charged defects provided the lowest energy configurations. The isolated anti-sites were considered with varying charges, and it was found that when removing 2 electrons from the system for magnesium and 3 for aluminium, the formation energy was at least 6 $\mathrm{eV}$ less than when using alternative integer charges of up to 4.

Defects considered here are isolated and neighbouring anti-sites, interstitials, split interstitials and the aluminium ring defect comprised of three aluminium interstitials and three aluminium vacancies. These defects were frequently observed in empirical potential based cascade simulations ${ }^{1,2}$. Formation energies for these point defects were considered in the standard way, as described for the simpler $\mathrm{MgO}$ system in our previous work ${ }^{11}$. This involves computing the total energy of a relaxed supercell containing the defect and the energy of a perfect supercell of the same quantity of atoms. The latter value is subtracted from the former value in order to compute the formation energy for the considered defect.

In addition to computing structures and energetics of these isolated point defects, clusters of such defects were also studied using the DFT methodology. Binding energies of clusters in a 224 atom cell were computed using the following equation,

$$
E_{\text {binding }}^{n}=E_{\text {cluster }}^{n}-\sum_{i=1}^{m} E_{i}^{224}+(224 m-n) E_{\text {perfect }}^{\text {atom }}
$$

where $n$ is the number of atoms in the cell containing the cluster, $m$ is the number of point defects comprising the cluster, $E_{\text {cluster }}^{n}, E_{i}^{224}$ and $E_{\text {perfect }}^{\text {atom }}$ are energies of the cell containing the defect cluster, the cell containing one of the isolated defects and the energy per atom in a perfect cell. To ensure convergence of the binding energy as a function of system size, different values of $n$ were chosen consistent with the ability to perform the calculations within a reasonable computing time.

DFT calculations were compared to empirical potential approximations which utilise a standard Buckingham potential $^{20}$ for interactions between atoms situated less than $8 \AA$ apart (this is given in Eq. 2, with $V(r)$ representing the Coulomb potential). Interactions in the remainder of the system are modelled by the Coulomb interaction alone. The parameters $A, C$ and $\rho$ have been quoted in previous work by Smith et al. ${ }^{1}$. 


$$
\phi(r)=A \exp \left(-\frac{r}{\rho}\right)-\frac{C}{r^{6}}+V(r)
$$

The empirical potential uses a full fixed charge model with charges of $+2,+3$ and -2 on $\mathrm{Mg}$, $\mathrm{Al}$ and $\mathrm{O}$ respectively. A Bader charge analysis using DFT gives values of 1.78, 2.56 and -1.73 .

\section{RESULTS}

A common defect in spinel is the cation exchange known as an anti-site defect. The formation energies of pairs of isolated anti-sites were computed using three different supercell sizes. Table I shows the computed formation energies. From this it can be seen that the formation energy of the anti-site defect pair converges to approximately 0.64 eV.

TABLE I: The formation energies from the DFT calculations for the pairs of isolated anti-sites as a function of the system size.

\begin{tabular}{cc}
\hline Number of Atoms Formation Energy $(\mathrm{eV})$ \\
\hline 56 & 0.55 \\
112 & 0.65 \\
224 & 0.64 \\
\hline
\end{tabular}

Neighbouring anti-sites were also considered so systems were created that contained pairs of anti-sites. The supercells used in these calculations were optimised to reduce the interaction between the actual defect and images produced by the periodic boundary conditions. Table II shows the formation energies for nearest neighbour anti-site defects ranging from first to fifth nearest neighbour. The DFT results presented are the values computed using the largest supercell size for each defect.

TABLE II: The formation energies for pairs of neighbouring anti-site defects

\begin{tabular}{|c|c|c|c|}
\hline \multirow[t]{2}{*}{ Anti-site Separation } & \multicolumn{3}{|c|}{ Formation Energy $(\mathrm{eV})$} \\
\hline & DFT & Empirical Potentials (without shells) & Empirical Potentials (with shells) \\
\hline $1 \mathrm{NN}(3.4 \AA)$ & 0.48 & 0.94 & 0.98 \\
\hline $2 \mathrm{NN}(5.3 \AA)$ & 0.67 & 1.00 & 0.98 \\
\hline $3 \mathrm{NN}(6.6 \AA)$ & 0.68 & 1.23 & 1.21 \\
\hline $4 \mathrm{NN}(7.8 \AA)$ & 0.75 & 1.26 & 1.23 \\
\hline $5 \mathrm{NN}(8.8 \AA)$ & 0.77 & 1.30 & 1.25 \\
\hline Isolated & 0.64 & 1.50 & $1.46^{21}$ \\
\hline
\end{tabular}

Both methodologies predict an increase in formation energy as a function of increasing anti-site separation up to the 5th nearest neighbour. However, in the case of the empirical potential model the formation energy continues to increase monotonically whereas in the DFT case, the energy reduces slightly after the 5th nearest neighbour position. Due to the very small magnitude of these anti-site formation energies, convergence of the energies to a satisfactory degree is difficult to obtain using DFT and hinders a more accurate assessment. Calculations were not possible for 6th nearest neighbour positions due to the large cell size required. It can be seen that the empirical potentials overestimate the absolute value of the formation energies of interacting anti-site pairs by a value between $0.4 \mathrm{eV}$ and $0.7 \mathrm{eV}$.

In addition to anti-site defects, isolated interstitial and vacancy defects ${ }^{1,2}$ can also form following a collision cascade. Using the same DFT methodology, the structure and energetics of different interstitial defects have been studied. The structures in Fig. 1 show two different possible configurations of a system containing a magnesium interstitial atom. It is found using empirical potentials that the split interstitial structure shown in Fig. 1 (b) is the more favourable. This split-interstitial consists of magnesium interstitials sitting near two unoccupied octahedral lattice sites around a magnesium vacancy. Values of the displacements of the neighbouring atoms for both structures are displayed in Table III and are taken from the DFT simulation from a large 224 atom supercell. 
TABLE IV: Formation energies (in eV) of the magnesium interstitial defects shown in Fig. 1 computed with DFT using a variety of supercell sizes. The computation of these values involved combined the magnesium interstitial structure with an isolated magnesium vacancy. Also included are formation energies computed using the empirical potential.

\begin{tabular}{ccc}
\hline Number of Atoms & Structure I Structure II \\
\hline 56 & 5.01 & 6.30 \\
112 & 7.05 & 6.36 \\
168 & 7.56 & 6.56 \\
224 & 7.58 & 6.68 \\
\hline E.P. (With Shells) & 11.4 & 10.9 \\
E.P. (Without Shells) & 12.8 & 12.4 \\
\hline
\end{tabular}

empirical potentials also favour structure II but the formation energies are almost double those computed using DFT. There is also less relative difference between the two. Structure I was never observed in the cascade simulations using empirical potentials. It can also be seen from Table IV that that the 56 atom cell is too small to give accurate formation energies. This is also in agreement with the previous calculations concerning antisites shown in table I.

The aluminium interstitials have also been investigated. A single aluminium interstitial atom placed on an octahedral site as shown in Fig. 2 (a), produces a very high formation energy of over $9.7 \mathrm{eV}$. A more favourable structure is shown in Fig. 2 (b), which consists of a magnesium-aluminium split interstitial around a magnesium vacancy. This is found to have a formation energy of approximately $8.4-8.8 \mathrm{eV}$ computed using DFT. In this calculation a corresponding isolated aluminium vacancy was combined with the aluminium interstitial structures when computing their formation energies. Empirical potentials again overestimate this value, predicting that the split interstitial defect has a formation energy of $13.9 \mathrm{eV}$ using a model with shells (and $15.1 \mathrm{eV}$ without shells). The difference in value between the formation energy of the $\mathrm{Mg}-\mathrm{Mg}$ split interstitial and the $\mathrm{Al}-\mathrm{Mg}$ split interstitial of around $2 \mathrm{eV}$ suggests that the latter would likely decay into the $\mathrm{Mg}-\mathrm{Mg}$ split together with a $\mathrm{Al}$ antisite defect although, using DFT, we have not calculated the transition energy barrier for this to happen or the formation energy of this combined cluster, due to the large cell size required to perform the calculation. However empirical potential simulations using temperature accelerated dynamics predict that this occurs with an energy barrier of $0.56 \mathrm{eV}^{3}$, this explains why the $\mathrm{Al}-\mathrm{Mg}$ split interstitial is rarely observed in classical MD simulations for this system. The ab-initio barrier height may disagree with the empirical potential barrier but from the results it is clear that both predicted the $\mathrm{Mg}-\mathrm{Mg}$ split interstitial with an $\mathrm{Al}$ antisite defect to be preferable to the $\mathrm{Al}-\mathrm{Mg}$ split interstitial.

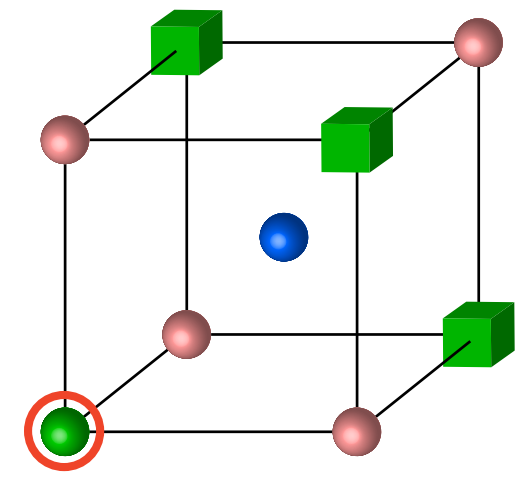

(a)

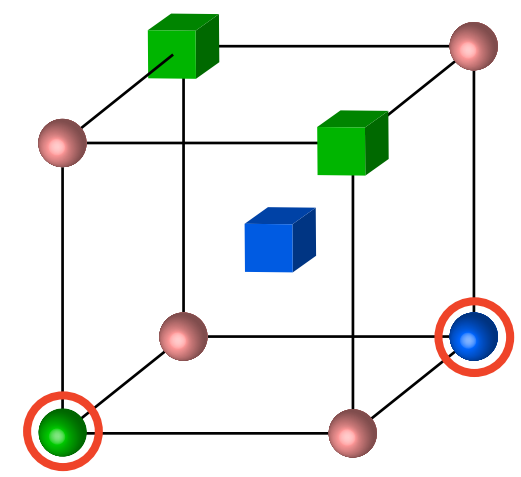

(b)

FIG. 2: Two possible aluminium defect structures in spinel. Structure I in figure (a) consists of a single aluminium interstitial on an octahedral structural vacancy and structure II in figure (b) consists of a magnesium-aluminium split interstitial and a magnesium vacancy.

A variation of the magnesium-aluminium interstitial structure was also considered, in which the aluminium interstitial atom is situated on the magnesium site (forming an antisite) instead of on an octahedral site. DFT found the formation energy of this configuration to be $9.5-9.6 \mathrm{eV}$ thus having a higher formation energy than the structure shown in Fig. 2(b). This is in agreement with the predictions obtained using empirical potential simulations. Isolated 
or split aluminium-aluminium interstitials were not found in empirical potential simulations and when carrying out the DFT computations for a split aluminium-aluminium di-interstitial, it was found that these have a large formation energy of $17.2 \mathrm{eV}$. Thus the empirical and DFT calculations are in agreement with the type of Al interstitial defect that forms if not with the exact values of the formation energy.

The final interstitial defect considered is that of oxygen. Forming an oxygen split-interstitial in a $<110>$-type direction over a vacant oxygen site is possible in several different configurations. It was found that only two of these are unique, due to the symmetry properties of the lattice. These structures have been reported previously in empirical potential simulations ${ }^{3}$. Two unique configurations are shown in Fig. 3 Computed formation energies are shown in

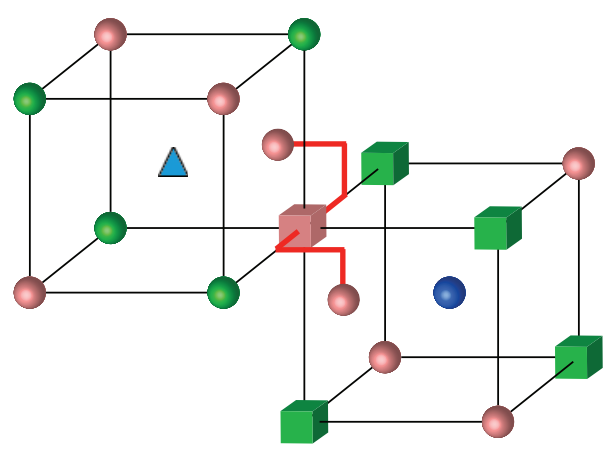

(a)

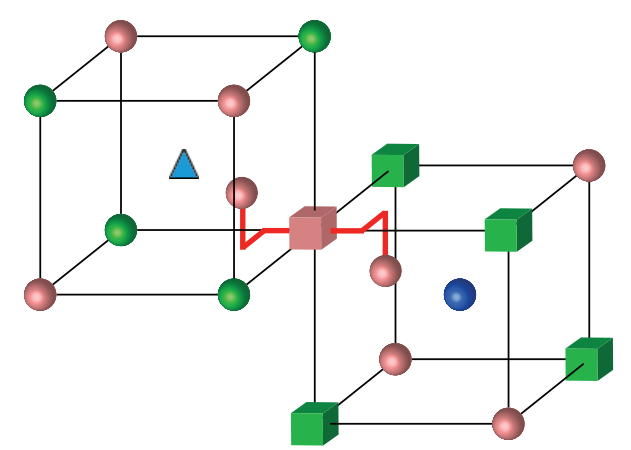

(b)

FIG. 3: Two possible oxygen split interstitial structures in $<110>$-type directions.

Table V. Although even larger supercell sizes are really required for the O interstitials, the formation energies are

TABLE V: Formation energies (in eV) of the oxygen split-interstitial configurations computed with DFT using a variety of supercell sizes

\begin{tabular}{ccc}
\hline Number of Atoms & Configuration (a) & Configuration (b) \\
\hline 56 & 8.74 & 9.51 \\
112 & 9.12 & 9.79 \\
224 & 8.86 & 9.41 \\
\hline
\end{tabular}

expected to converge, with respect to supercell size, to values in the region of $9.4-9.8$ eV for configuration (b) and $8.9-9.2 \mathrm{eV}$ for the more favourable structure, configuration (a). Empirical potential simulations overestimate this energy, predicting a value for the more favourable oxygen split-interstitial structure as 11.0 and $12.5 \mathrm{eV}$ for a model with and without shells respectively. However the geometry and relative stability of the two defect structures is the same with both DFT and the empirical potentials.

Another type of Al interstitial defect has been observed in collision cascade simulations. This is the so-called 'ring' defect, comprising of three aluminium interstitial atoms, occupying what were tetrahedral structural vacancy sites, and three aluminium vacancies in a circular formation. Due to the complexity of the defect, the DFT calculations become more demanding as it is difficult to reduce interactions between the defect and its periodic repeats. Nevertheless formation energies were calculated for two cell sizes, containing 112 atom and a 224 atoms. The resulting energies were found to be 7.66 and 7.34 respectively. Empirical potential simulations estimated a formation energy of approximately $10.2 \mathrm{eV}$, thus overestimating the energy by $2.5-3 \mathrm{eV}$.

In addition to considering simple point defects, we have also computed energetics of clusters of these simple defects. The calculated binding energies for dimer defects and single vacancy or interstitial defects bound to oppositely charged anti-site defects are shown in Table VI. Each of these binding energies was calculated using DFT using four different sized supercells for each defect to ensure convergence. Energies converged to approximately 2 decimal places for the smallest clusters and it is the value from the largest supercell that is displayed. Larger trimer clusters require larger supercells to ensure full convergence, energies displayed for these clusters have used supercells containing at least 280 atoms. Interstitials, vacancies and anti-site defects are denoted using the simplified Kröger-Vink notation ${ }^{22}$. The results in Tables VI and VII show that for each vacancy/interstitial the trimer cluster has a higher binding energy than the dimer. Generally first neighbour defect structures are formed and third neighbour configurations are never 
favoured. Comparing the binding energy of the clusters between the DFT and empirical potential results there are two cases where the ordering is different. In the case of the $\mathrm{Mg}$ vacancy and the $\mathrm{Al}$ antisite compared to the $\mathrm{Mg}$ vacancy and two $\mathrm{Al}$ antisites, the DFT calculations are so numerically close that it is impossible to distinguish which is really the most favourable. This is also the case for the oxygen-cation divacancy case.

All of the binding energies are negative, thus implying that defects are more likely to occur in clusters than as isolated. The trend of favourability is the same for the empirical potential simulations as reported by Ball et al. ${ }^{21}$. Generally the model with shells gives better agreement with the DFT calculations than the model without shells which shows a significant overestimation of the binding energies compared to DFT.

Ball et $a l .{ }^{21}$ give a methodology for examining defect reactions and hence relative concentrations of defects as a function of temperature based on a series of mass action equations. We have used DFT to compute the same process energies that have previously been calculated by Ball et al. and these energies are presented in tables VI and VII. We have considered isolated defects along with dimer and trimer clusters. Larger clusters are unfeasible due to the large supercells that would be required to obtain convergence of formation energy.

These results indicate that in general, both methodologies predict the aluminium defects to have the highest energies, when compared to similar defects comprising of the other species. All results here show that the isolated defects have larger energies than the dimers or trimers, in fact, the trimers are the lowest energy defect for each species. Magnitudes of energies are 150 to 200\% larger than DFT results when calculated using empirical potentials. The shell model produces the lower energies and so is more in line with DFT computations. DFT results predict that the Schottky trimer has the lowest energy of all non-isolated defects considered (with an energy of $2.77 \mathrm{eV}$ ), but empirical potential simulations indicate the lowest energy is that of oxygen trimer defects.

\section{CONCLUSIONS}

A main goal of this work was to determine if the defects observed at the end of cascade simulations using classical potentials were also the lowest energy structures predicted by DFT. We undertook this task by performing a series of ab-initio computations using PLATO and compared our findings with results generated from empirical potential simulations. We compared predicted structures of key defects (anti-sites, interstitials, and split-interstitials) along with their energetics.

Both methodologies predicted a low formation energy for anti-site defects. Naturally occurring spinel has been shown to contain large numbers of these defects and thus it is no surprise that their formation energy is low. The empirical potential for spinel always gives higher formation energies than DFT. This is found to be true regardless of the separation distance between the two anti-sites comprising the pair. Anti-site formation energies increase as the pairs of anti-site atoms become more separated, with formation energies for first nearest neighbours of anti-sites showing the highest favourability of all considered pairs.

Two configurations of magnesium interstitial atoms (shown in Fig. 1) were studied using DFT. Both of these initial configurations, when relaxed, were found to form a split-interstitial around the vacant magnesium site. It was found that the lowest formation energy (of less than $6.7 \mathrm{eV}$ ) came from structure II, which might also be thought of as a representing a partial rocksalt-like transformation with magnesium atoms sitting near octahedral previously unoccupied sites. These are on opposite corners of a smaller cube which also comprises of oxygen atoms.

Aluminium interstitials were found to be unfavourable when configurations consisted of either isolated or split aluminium-aluminium interstitials (DFT computed formation energies of over $9.7 \mathrm{eV}$ and $17.2 \mathrm{eV}$ for each of these respectively). It was found that a split aluminium-magnesium interstitial had the more favourable structure with a formation energy of less than $8.8 \mathrm{eV}$. This is approximately $2 \mathrm{eV}$ higher than the lowest formation energy of any magnesium split interstitial structure. Few aluminium interstitials were found in cascade simulations, but when they were, they formed either a magnesium-aluminium split interstitial or a more complicated ring defect, which comprised of three aluminium interstitials and three aluminium vacancies. We studied this ring defect using DFT and found the formation energy to be $7.34 \mathrm{eV}$. The empirical potential model predicts a formation energy of $10.2 \mathrm{eV}$.

Empirical potential simulations have previously indicated that a more favourable structure to a $\mathrm{Mg}$ - $\mathrm{Al}$ split interstitial is a $\left\{\mathrm{Al}_{\mathrm{Mg}}: \mathrm{Mg}_{\mathrm{i}}-\mathrm{V}_{\mathrm{Mg}}-\mathrm{Mg}_{\mathrm{i}}\right\}$ cluster. Our work using DFT has verified that this cluster is expected to have a lower energy than the aforementioned $\mathrm{Al}-\mathrm{Mg}$ split interstitial. This conclusion is reached by observing the formation energy of both the isolated anti-site and formation energy for the $\mathrm{Mg}-\mathrm{Mg}$ split interstitial.

The general trend is that empirical potentials overestimate the formation energies computed using the ab-initio methodology, but correctly assess the relative favourability of defect structures. A possible reason for this might be that the Coulomb interactions using the full fixed charge model is too strong. Bader charge analysis indicates values that are $11-15 \%$ less.

The magnitudes of binding energies of clustered defects were overestimated by up to 3-4 times with empirical potential simulations but, again, the order of favourability of the magnitudes is similar. Both methodologies agree 
that defects in clusters are preferable to their isolated counterparts. The implications for cascade simulations are that the larger binding energies predicted for the empirical potentials imply that the number of defects produced may well be underestimated compared to experiment.

\section{ACKNOWLEDGEMENTS}

C. A. Gilbert thanks Loughborough University for providing funding. DFT computations were performed using facilities provided by the Loughborough High Performance Computing Centre. We would also like to acknowledge discussions with Blas Uberuaga and Kurt Sickafus.

1 R. Smith, D. Bacorisen, B. P. Uberuaga, K. E. Sickafus, J. A. Ball, and R. W. Grimes, J. Phys.: Condens. Matter 17, 875 (2005).

2 D. Bacorisen, R. Smith, B. P. Uberuaga, K. E. Sickafus, J. A. Ball, and R. W. Grimes, Phys. Rev. B 74, 214105 (2006).

3 B. P. Uberuaga, D. Bacorisen, R. Smith, J. A. Ball, R. W. Grimes, A. F. Voter, and K. E. Sickafus, Phys. Rev. B 75, 104116 (2007).

${ }^{4}$ K. E. Sickafus, A.C.Larson, N.Yu, M. Nastasi, G. W. Hollenberg, F. A. Garner, and R. Bradt, J. Nucl. Mater. 219, 128 (1995).

5 N. Bordes, L. M. Wang, R. Ewing, and K. E. Sickafus, J. Mater. Res. 10, 981 (1995).

6 M. Ishimaru, I. V. Afanasyev-Charkin, and K. E. Sickafus, Appl. Phys. Lett. 76, 2556 (2000).

7 N. Yu, K. E. Sickafus, and M. Nastasi, Phil. Mag. Lett. 70, 235 (1994).

8 M. T. Warren, M. T. Dove, and S. A. T. Redfern, J. Phys.: Condens. Matter 12, L43 (2000).

9 J. A. Ball, M. Pirzada, R. W. Grimes, M. O. Zacate, D. W. Price, and B. P. Uberuaga, J. Phys.: Condens. Matter 17, 7621 (2005).

10 H. Moriwake, I. Tanaka, F. Oba, Y. Koyama, and H. Adachi, Phys. Rev. B 65, 153103 (2002).

11 C. A. Gilbert, S. D. Kenny, R. Smith, and E. Sanville, Phys. Rev. B 76, 184103 (2007).

12 S. D. Kenny, A. P. Horsfield, and H. Fujitani, Phys. Rev. B 62, 4899 (2000).

13 W. Kohn and L. J. Sham, Phys. Rev. 140, A1133 (1965).

14 P. Hohenberg and W. Kohn, Phys. Rev. 136, B864 (1964).

15 S. Goedecker, M. Teter, and J. Hutter, Phys. Rev. B 54, 1703 (1996).

16 C. Hartwigsen, S. Goedecker, and J. Hutter, Phys. Rev. B 58, 3641 (1998).

17 R. P. Gupta, J. Nucl. Mater. 358, 35 (2006).

18 V. R. Saunders, R. Dovesi, C. Roetti, R. Orlando, C. M. Zicovich-Wilson, N. M. Harrison, K. Doll, B. Civalleri, I. Bush, P. D'Arco, et al., CRYSTAL2003 User's Manual (University of Torino, 2003).

19 K. E. Sickafus, J. M. Mills, and N. W. Grimes, J. Am. Ceram. Soc. 82, 3279 (1999).

${ }^{20}$ R. A. Buckingham, Proc. R. Soc. London, Ser. A 168, 264 (1938).

21 J. A. Ball, S. T. Murphy, R. W. Grimes, D. Bacorisen, R. Smith, B. P. Uberuaga, and K. E. Sickafus, Solid State Sciences 10, 717 (2008).

22 F. A. Kröger, The Chemistry of Imperfect Crystals (North Holland Publishing Company Ltd., 1974). 
TABLE VI: Binding energies of defect clusters computed using both DFT and empirical potential simulations.

\begin{tabular}{|c|c|c|c|}
\hline \multirow[t]{2}{*}{ Cluster } & \multicolumn{3}{|c|}{ Binding Energy $(\mathrm{eV})$} \\
\hline & DFT & Empirical Potential (shells) & Empirical Potential (no shells) \\
\hline$\left\{\ddot{V}_{\mathrm{O}}: \mathrm{Mg}_{\mathrm{Al}}^{\prime}\right\}^{\cdot}$ & -0.57 & -1.55 & -1.85 \\
\hline$\left\{\mathrm{V}_{\mathrm{O}}: 2 \mathrm{Mg}_{\mathrm{Al}}^{\prime}\right\}^{\times}$ & -0.89 & -2.49 & -3.01 \\
\hline$\left\{\mathrm{V}_{\mathrm{Mg}}^{\prime \prime}: \mathrm{Al}_{\mathrm{Mg}}\right\}^{\prime}$ & -0.1 & -0.81 & -0.91 \\
\hline$\left\{\mathrm{V}_{\mathrm{Mg}}^{\prime \prime}: 2 \mathrm{Al}_{\mathrm{Mg}}\right\}^{\times}$ & -0.0025 & -1.39 & -1.54 \\
\hline$\left\{\mathrm{V}_{\mathrm{A} 1}^{\prime \prime \prime}: \mathrm{Al}_{\mathrm{Mg}}\right\}^{\prime \prime}$ & -0.36 & -1.45 & -1.72 \\
\hline$\left\{\mathrm{V}_{\mathrm{Al}}^{\prime \prime \prime}: 2 \mathrm{Al}_{\mathrm{Mg}}\right\}^{\prime}$ & -0.89 & -2.61 & -3.20 \\
\hline$\left\{\mathrm{Al}_{\mathrm{Mg}}: \mathrm{Mg}_{\mathrm{A} 1}^{\prime}\right\}^{\times}$ & -0.15 & -0.48 & -0.60 \\
\hline$\left\{\mathrm{O}_{i}^{\prime \prime}: \mathrm{Al}_{\mathrm{Mg}}\right\}^{\times}$ & -0.83 & -1.73 & -2.36 \\
\hline$\left\{\mathrm{O}_{i}^{\prime \prime}: 2 \mathrm{Al}_{\mathrm{Mg}}\right\}^{\prime}$ & -1.32 & -2.82 & -4.57 \\
\hline$\left\{\mathrm{Mg}_{i}: \mathrm{Mg}_{\mathrm{Al}}^{\prime}\right\}^{\cdot}$ & -0.36 & -1.04 & -1.24 \\
\hline$\left\{\mathrm{Mg}_{i}^{\ddot{*}}: 2 \mathrm{Mg}_{\mathrm{Al}}^{\prime}\right\}^{\times}$ & -0.67 & -1.73 & -2.1 \\
\hline$\left\{\mathrm{Al}_{i}^{\cdots}: \mathrm{Mg}_{\mathrm{Al}}^{\prime}\right\}^{*}$ & -0.44 & -1.51 & -1.82 \\
\hline$\left\{\mathrm{Al}_{i}^{\cdots}: 2 \mathrm{Mg}_{\mathrm{Al}}^{\prime}\right\}^{\cdot}$ & -0.83 & -2.59 & -3.01 \\
\hline$\left\{\mathrm{Al}_{i}^{\cdots}: \mathrm{O}_{i}^{\prime \prime}\right\}^{\cdot}$ & -1.28 & -3.53 & -5.44 \\
\hline$\left\{\mathrm{Mg}_{i}: \mathrm{O}_{i}^{\prime \prime}\right\}^{\times}$ & -0.32 & -1.91 & -2.17 \\
\hline$\left\{\mathrm{V}_{\mathrm{O}}: \mathrm{V}_{\mathrm{Mg}}^{\prime \prime}\right\}^{\times}$ & -2.04 & -4.06 & -4.57 \\
\hline$\left\{\mathrm{V}_{\mathrm{O}}: \mathrm{V}_{\mathrm{Al}}^{\prime \prime \prime}\right\}$ & -2.01 & -4.67 & -5.35 \\
\hline
\end{tabular}

TABLE VII: Energies for defect processes. These values have been normalised per defect formed.

\begin{tabular}{ccccc}
\hline Process & Cluster & \multicolumn{3}{c}{ Energy (eV) } \\
\cline { 3 - 5 } & & DFT Empirical Potential (shells) & Empirical Potential (no shells) \\
\hline Mg Frenkel Isolated & 3.34 & 5.41 & 6.19 \\
Mg Frenkel & Dimer & 3.10 & 4.49 & 5.11 \\
Mg Frenkel & Trimer & 3.09 & 3.85 & 4.38 \\
\hline Al Frenkel & Isolated & 4.39 & 6.86 & 7.55 \\
Al Frenkel & Dimer & 3.98 & 5.38 & 5.78 \\
Al Frenkel & Trimer & 3.52 & 4.27 & 4.68 \\
\hline O Frenkel & Isolated & 4.43 & 5.49 & 6.25 \\
O Frenkel & Dimer & 3.73 & 3.82 & 4.14 \\
O Frenkel & Trimer & 3.33 & 2.84 & 2.84 \\
\hline Schottky & Isolated & 3.53 & 5.32 & 5.99 \\
Schottky & Dimer & 3.08 & 3.90 & 3.31 \\
Schottky & Trimer & 2.77 & 2.95 & - \\
\hline Anti-site & Isolated & 0.64 & 0.73 & 3.29 \\
\hline
\end{tabular}

